\title{
Association between supportive interventions and healthcare utilization and outcomes in patients on long-term prescribed opioid therapy presenting to acute healthcare settings: a systematic review and meta-analysis
}

\author{
Jean Deschamps ${ }^{1 *}$ (D) James Gilbertson ${ }^{2}$, Sebastian Straube ${ }^{3}$, Kathryn Dong $^{4}$, Frank P. MacMaster ${ }^{5}$,
} Christina Korownyk ${ }^{6}$, Lori Montgomery ${ }^{7}$, Ryan Mahaffey ${ }^{8}$, James Downar ${ }^{9}$, Hance Clarke ${ }^{10,11}$, John Muscedere ${ }^{12}$, Katherine Rittenbach ${ }^{13,14,15}$, Robin Featherstone ${ }^{16}$, Meghan Sebastianski ${ }^{17}$, Ben Vandermeer ${ }^{16}$, Deborah Lynam ${ }^{18}$, Ryan Magnussen ${ }^{19}$, Sean M. Bagshaw ${ }^{1}$ and Oleksa G. Rewa ${ }^{1}$

\footnotetext{
Abstract

Background: Long-term prescription of opioids by healthcare professionals has been linked to poor individual patient outcomes and high resource utilization. Supportive strategies in this population regarding acute healthcare settings may have substantial impact.

Methods: We performed a systematic review and meta-analysis of primary studies. The studies were included according to the following criteria: 1) age 18 and older; 2) long-term prescribed opioid therapy; 3) acute healthcare setting presentation from a complication of opioid therapy; 4) evaluating a supportive strategy; 5) comparing the effectiveness of different interventions; 6 ) addressing patient or healthcare related outcomes. We performed a qualitative analysis of supportive strategies identified. We pooled patient and system related outcome data for each supportive strategy.

\footnotetext{
*Correspondence: jean3@ualberta.ca

'Department of Critical Care Medicine, Faculty of Medicine and Dentistry, University of Alberta, 8440112 St. NW, Critical Care Medicine 2-124E Clinical Sciences Building, Edmonton, Alberta T6G 2B7, Canada

Full list of author information is available at the end of the article
}

C C The Author(s). 2021 Open Access This article is licensed under a Creative Commons Attribution 4.0 International License, which permits use, sharing, adaptation, distribution and reproduction in any medium or format, as long as you give appropriate credit to the original author(s) and the source, provide a link to the Creative Commons licence, and indicate if changes were made. The images or other third party material in this article are included in the article's Creative Commons licence, unless indicated otherwise in a credit line to the material. If material is not included in the article's Creative Commons licence and your intended use is not permitted by statutory regulation or exceeds the permitted use, you will need to obtain permission directly from the copyright holder. To view a copy of this licence, visit http://creativecommons.org/licenses/by/4.0/. The Creative Commons Public Domain Dedication waiver (http://creativecommons.org/publicdomain/zero/1.0/) applies to the data made available in this article, unless otherwise stated in a credit line to the data. 
(Continued from previous page)

Results: A total of 5664 studies were screened and 19 studies were included. A total of 9 broad categories of supportive strategies were identified. Meta-analysis was performed for the "supports for patients in pain" supportive strategy on two system-related outcomes using a ratio of means. The number of emergency department (ED) visits were significantly reduced for cohort studies $\left(n=6,0.36,95 \% \mathrm{Cl}[0.20-0.62], \mathrm{I}^{2}=87 \%\right)$ and randomized controlled trials (RCTs) $\left(n=3,0.71,95 \% \mathrm{Cl}[0.61-0.82], \mathrm{I}^{2}=0 \%\right)$. The number of opioid prescriptions at ED discharge was significantly reduced for $\mathrm{RCTs}\left(n=3,0.34,95 \% \mathrm{Cl}[0.14-0.82], \mathrm{I}^{2}=78 \%\right)$.

Conclusion: For patients presenting to acute healthcare settings with complications related to long-term opioid therapy, the intervention with the most robust data is "supports for patients in pain".

Keywords: Opioid, Addiction medicine, Substance-related disorders, Drug abuse, Hospital medicine

\section{Background}

\section{Description of the condition}

The opioid epidemic is a major public health problem across the world. While not initially recognized as a crisis, it has become a public health emergency that is largely believed to have begun in 1996 when OxyContin ${ }^{\circ}$ was approved by the FDA [1]. Opioid prescription was initially thought to be the crux of the problem and multiple interventions were implemented in response over the years. Most of these interventions were focused on reducing access to opioid prescriptions with unforeseen and paradoxical consequences. Indeed, strictly targeting a reduction in opioid access ignored the complex socio-economic factors that impact opioidrelated morbidity and mortality. As an example, reductions in prescription rates in Ontario (Canada) lead to a doubling of opioid mortality due to an increase in street opioids [2]. This shift in the nature of the opioid crisis to illicit opioid use has indeed been seen in multiple locations over the years and paralleled these interventions [3]. Considering such findings, illicit drug use and harm reduction related to their use has become the focus of most current efforts to limit the impact of the opioid crisis and the impact of opioid prescriptions has taken a backseat. However, it remains that there are patients on long-term opioids for chronic painful conditions without diagnosed or suspected opioid use disorder that are also at risk for complications. This group has ultimately been somewhat neglected amidst the extensive literature available on the more obvious high-risk populations. It is however quite clear that individuals on long-term opioid therapy are at risk of poor outcomes, including hospitalization, overdose and death from the use or management of their opioids $[4,5]$. These poor outcomes invariably lead to acute healthcare presentations that have significant system level impacts on health services use including emergency department (ED) presentations, hospital and intensive care unit (ICU) admissions, as well important socioeconomic consequences [6-10].

\section{Description of the intervention}

Harm reduction strategies are defined as "any policy or program designed to reduce drug-related harm without requiring the cessation of drug use; these interventions may be targeted at the individual, the family, community or society [11-19]." Harm reduction strategies are traditionally thought of and applied to patients demonstrating high risk behaviors and the term has not typically been applied in relation to individuals on long-term opioid therapy without a diagnosed or suspected opioid use disorder. These individuals may still benefit from harm reduction strategies as they require these medications for their well-being but may still suffer harmful consequences from their use. Harm reduction strategies are however traditionally associated with opioid use disorder. There is thus ample evidence for harm reduction strategies in acute opioid overdoses that is lacking for individuals on long-term opioid therapy without opioid use disorder. Their impact may be substantial given the reported high risk of adverse events in these settings [7, 20-22]. As the strategies applying to this population may be different, we will instead refer to them as "supportive interventions" to limit confusion with strategies associated with opioid use disorder.

The most important complications as a result of opioid therapy most often lead to a presentation to an acute healthcare setting, defined as a setting in which "health system components, or care delivery platforms, are used to treat sudden, often unexpected, urgent or emergent episodes of injury and illness that can lead to death or disability without rapid intervention [23]." Examples of these include emergency departments, acute health care clinics, and hospital inpatient units. Accordingly, this may be the healthcare setting in which the most impactful supportive interventions may lie, and how this population can best be captured in studies. As such, the primary objective of this study was to identify the most effective supportive interventions for patients on longterm prescribed opioids presenting to acute care settings to decrease complications attributable to opioid use, to reduce avoidable health services use, and to improve outcomes. 


\section{Methods}

\section{Study design and registration}

We performed a systematic review using the guidelines from Cochrane and the Centre for Reviews and Dissemination [24, 25], and reported according to the Preferred Reporting Items for Systematic Reviews and Metaanalyses (PRISMA) guideline and the Meta-analysis Of Observational Studies in Epidemiology (MOOSE) guidelines for observational studies [26, 27]. The study was registered with the PROSPERO (CRD42018088962 on 2018/02/19) International Prospective Register of Systematic Reviews (http://ww.crd.york.ac.uk/prospero).

\section{Criteria for considering studies for this review Types of studies}

We considered primary studies (i.e., randomized controlled trials (RCT), cohort studies, case-control studies) and secondary analyses or evidence syntheses (i.e., systematic reviews, meta-analyses). There were no language restrictions. We considered studies published after 1996 as this is when OxyContin ${ }^{\circ}$ was introduced, and the current prescription opioid epidemic is believed to have largely began [1]. We excluded editorials, case series, case reports and narrative reviews.

\section{Eligibility of individual studies}

Studies were eligible for inclusion if they satisfied the following criteria:

- Patient related criteria:

- Age 18 years or older.

- Long-term opioid therapy, reflecting prescribed opioid more than $70 \%$ of days for at least 3 months [28].

- Presentation to acute healthcare setting secondary for a presumed or confirmed complication of prescribed opioid therapy.

- Study-related criteria

- Evaluating an intervention representing a harm reduction strategy.

- Comparing the effectiveness of different interventions between each other or individual interventions compared to current care.

- Addressing patient or healthcare system related outcomes (i.e. number of opioid prescriptions, repeat presentations to ED or acute healthcare, number of overdoses).

We excluded studies that specifically addressed patients with non-prescription opioid use, or prescription opioid use not obtained through healthcare professionals. We excluded studies of patients with opioid use disorder or misuse that was not attributable to an established chronic pain disorder or disease. These exclusions were chosen to target patients requiring long-term recurrent opioid use for medical conditions without a diagnosed or suspected disorder. Additionally, we eschewed the more stringent definition of long-term or chronic opioid therapy which usually is an "episode duration of $90+$ days and 10+ opioid prescriptions or $120+$ days supply of opioids dispensed [29]." We used a less stringent criteria to account for an expected smaller sample of population given the exclusions as mentioned. We will thus avoid the term "chronic opioid therapy" and use "long-term opioid therapy" as the former has stronger association with opioid use disorder and this may result in confusion for the reader.

\section{Search methods}

The search strategy was developed and executed by an information specialist (RF) and was peer-reviewed by a second research librarian (Table 1). The information specialist searched electronic databases: Ovid MEDLINE (1946-), Ovid EMBASE (1996-), Cumulative Index to Nursing and Allied Health Literature (CINAHL) via EBSCOhost (1937-), and Wiley Cochrane Library (inception-), including the Cochrane Database of Systematic Reviews and the Cochrane Central Register of Controlled Trials (CENTRAL). We also searched reports from the National Information Center on Health Services Research and Health Care Technology (NICHSR) via the NICHSR ONESearch portal. Study records were also searched via the trial registry platform ClinicalTrials.gov, guidelines via the National Guidelines Clearinghouse, and meeting abstracts via the Conference Proceedings Citation Index database (Clarivate Analytics). The following search themes was used: 1) opioids; 2) long-term drug therapy; and 3) acute healthcare settings (emergency departments, acute care surgery, critical care, urgent care and short-term inpatient stabilization). We additionally scanned the reference list of relevant included studies for additional articles. Bibliographic records were exported to an EndNote X9 (Clarivate Analytics, Philadelphia, Pennsylvania). Databases were searched up to and including April 11, 2019.

\section{Study assessment}

Articles were identified through two phases. First, two authors (JD1 and JG) independently reviewed the titles and abstracts of all retrieved articles for study inclusion. Second, full texts of the selected articles were retrieved, reviewed and selected based on inclusion criteria. All steps were performed in duplicate, and in each phase, disagreements were resolved through discussion. In the case of unresolved matters, a third author (OGR) was involved. Reasons for exclusion of full text articles were recorded and displayed in a PRISMA diagram format. 
Table 1 Search strategy

\section{Database: Ovid MEDLINE(R) Epub Ahead of Print, In-Process \& Other Non-Indexed Citations, Ovid MEDLINE(R) Daily and Ovid MEDLINE(R)} 1946 to Present

1 exp. Narcotics/ (111132)

2 actiq*.tw,kf. (27)

3 carfentan*.tw,kf. (243)

4 codeine ${ }^{*}$. tw, kf. (4872)

5 demerol*.tw,kf. (231)

6 (dihydro-morph* or dihydromorph*).tw,kf. (451)

7 dilaudid $^{*}$. tw,kf. (69)

8 dur?gesic*.tw,kf. (84)

9 fentanyl*.tw,kf. (16667)

10 fentora*.tw,kf. (9)

11 heroin.tw,kf. (12893)

12 (hydro-codone* or hydrocodone*).tw,kf. (858)

13 (hydro-morphone* or hydromorphone*).tw,kf. (1359)

14 morphine*.tw,kf. (47330)

15 narcotic*.tw,kf. (14412)

16 lorcet* $^{*} . \mathrm{tw}, \mathrm{kf} .(5)$

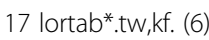

18 opiate $^{*}$. tw,kf. (23769)

19 opioid* $^{*}$.tw,kf. (73603)

20 (oxy-codone* or oxycodone*).tw,kf. (2670)

21 (oxy-contin* or oxycontin*).tw,kf. (226)

22 percocet*.tw,kf. (58)

23 percodan*.tw,kf. (14)

24 pethidine ${ }^{*} . t w, k f .(2304)$

25 phentanyl*.tw,kf. (119)

26 sublimaze*.tw,kf. (22)

27 vicodin*.tw,kf. (56)

28 or/1-27 [Combined MeSH \& text words for opioids] (186296)

29 Addiction Medicine/ (4)

30 Behavior, Addictive/ (7744)

31 exp. "Chemical and Drug Induced Liver Injury"/ (26678)

32 Drug abuse/ (87805)

33 exp. Drug Misuse/ (10703)

34 Drug Overdose/ (9457)

35 Neurotoxicity Syndromes/ (4428)

36 exp. Opioid-Related Disorders/ (22304)

37 Poisoning/ (21631)

38 Psychoses, Substance-Induced/ (5082)

39 Self-Injurious Behavior/ (6447)

40 Street Drugs/ae [adverse effects] (1421)

41 Substance-Related Disorders/ (87805)

42 Substance Withdrawal Syndrome/ (20325)

43 ((abus* or addict* or chronic* or depend* or disorder* or intoxicat* or mis-us* or misus* or over-dos* or overdos* or poison* or withdrawal*) adj3 (drug* or fentanyl* $^{*}$ or heroin* or narcotic* or opiate* or opioid* or oxy-co* or oxyco* or morphine*)).tw,kf. (97309) 
Table 1 Search strategy (Continued)

\section{Database: Ovid MEDLINE(R) Epub Ahead of Print, In-Process \& Other Non-Indexed Citations, Ovid MEDLINE(R) Daily and Ovid MEDLINE(R)} 1946 to Present

44 ((drug* or substance* or toxic*) adj2 psycho*).tw,kf. (18771)

45 or/29-44 [Combined MeSH \& text words for chronic drug use] (263106)

46 Burn Units/ (2227)

47 Coronary Care Units/ (4202)

48 exp. Critical Care/ (51242)

49 Critical Care Nursing/ (1223)

50 Emergency Medicine/ (11989)

51 Emergency Nursing/ (6602)

52 exp. Perioperative Care/ and (acute* or critical* or emergenc* or intensiv* or trauma* or urgent*).mp. (19203)

53 Hospital Medicine/ (119)

54 exp. Hospitals/ and (acute* or critical* or emergenc* or intensiv* or trauma* or urgent*).mp. (42779)

55 Hospitalization/ (91123)

56 Intensive Care Units/ (45436)

57 exp. Life Support Care/ (8408)

58 Operating Rooms/ and (acute* or critical* or emergenc* or intensiv* or trauma* or urgent*).mp. (1581)

59 Respiratory Care Units/ (579)

60 exp. Specialties, Surgical/ and (acute* or critical* or emergenc* or intensiv* or trauma* or urgent*).mp. (16202)

61 Surgery Department, Hospital/ and (acute* or critical* or emergenc* or intensiv* or trauma* or urgent*).mp. (1066)

62 ((acute* or critical* $^{*}$ or emergenc* ${ }^{*}$ or intensiv* or trauma* or urgent*) adj2 (care or centr* or department* or hospital* or unit* or ward*)).tw,kf. (270869)

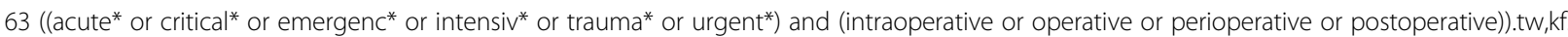
(114703)

64 ((burn* or cardi* or coronary* or heart* or respiratory*) adj2 (care or department* or room* or unit* or ward*)).tw,kf. (27819)

65 ICU.tw,kf. (44322)

66 life support.tw,kf. (10639)

67 or/46-66 [Combined MeSH \& text words for acute healthcare settings] (564082)

68 and/28,45,67 [Combined concepts for opioids, chronic drug use, \& acute healthcare settings] (2136)

69 exp. animals/ not humans/ (4426250)

7068 not 69 [Exclude animal studies] (2116)

71 (adolescent/ or exp. child/) not exp. adult/ (1302784)

72 (adolescen* or child* or infan* or neonat* or p?ediatric* or youth).ti,jw. (1500398)

7370 not (71 or 72) [Exclude pediatric studies] (1845)

74 (comment or editorial or news or newspaper article).pt. (1210379)

7573 not 74 [Exclude opinion pieces] (1810)

76 ("1996*" or "1997*" or "1998*" or "1999*" or $200^{*}$ or $201 *$ ).dt. (17023135)

77 and/75-76 [date limit applied] (1410)

78 limit 77 to (english or french) (1322)

79 remove duplicates from 78 (1315)

\section{Quality assessment of studies}

The methodological quality of each study was independently analyzed by two authors (JD1 and JG) using the Newcastle-Ottawa Quality Assessment Scale (NOS) for observational studies and the Cochrane Risk of Bias Tool for randomized controlled trials [24, 30]. Disagreements were resolved through discussion, and in the case of unresolved matters, a third author (OGR) was involved.

\section{Data analysis and synthesis}

An inventory of supportive strategies was developed from the included studies. Descriptive analysis and data 
extraction of patient and study characteristics, and of supportive strategies and their outcomes were performed using standardized electronic data forms (Supplementary Appendix 1 for variables extracted). We analyzed all available data qualitatively and, when possible, aggregate analysis was performed [31]. For each study we extracted or computed the ratio of means between the intervention and usual care groups with 95\% confidence interval [32]. These ratios were then pooled using the DerSimonian-Laird random effects method with an inverse variance weighting. To minimize bias due to confounding, results for RCTs were pooled separately from results for cohort studies and the two were not combined in one meta-analysis. Heterogeneity was assessed using the I-squared statistic with values greater than $50 \%$ considered to be "substantial" heterogeneity.

\section{Results}

Our search yielded 5664 studies, of which 21 studies fulfilled our eligibility criteria (Fig. 1, Supplementary Appendix 2). These included 17 full-text articles and 2 abstracts, of which 15 were cohort studies and 4 were randomized controlled trials (Table 2). No additional articles were identified for inclusion from the reference list of included articles. Study quality is reported in Tables 3 and 4 .

A total of 9 categories of supportive strategies were identified (Table 5). Most studies addressed multiple supportive strategies simultaneously making separation difficult. This is reflected and detailed in both Tables 2 and 5 . The 15 cohort studies included all assessed supportive strategies in a pre/post intervention model (Table 2). They either compared matched cohorts of different patients $(n=8)$ or cohorts of same patients before and after intervention $(n=7)$ in which each patient was his own control. Nearly all studies were performed in ED settings $(n=13)$. A single cohort study assessed mortality [11]. The four RCTs (Table 2) were all performed in the ED, with the comparator being usual care. Outcomes assessed included ED opioid prescriptions, ED discharge opioid prescriptions, hospital length of stay (LOS), and overdose and opioid related ED visits. All cohort and RCT studies were conducted in the US other than the cohort study by Allen et al. in Canada [36].

Six cohort studies and three RCTs were suitable for meta-analysis (Figs. 2 and 3). All studies included in the

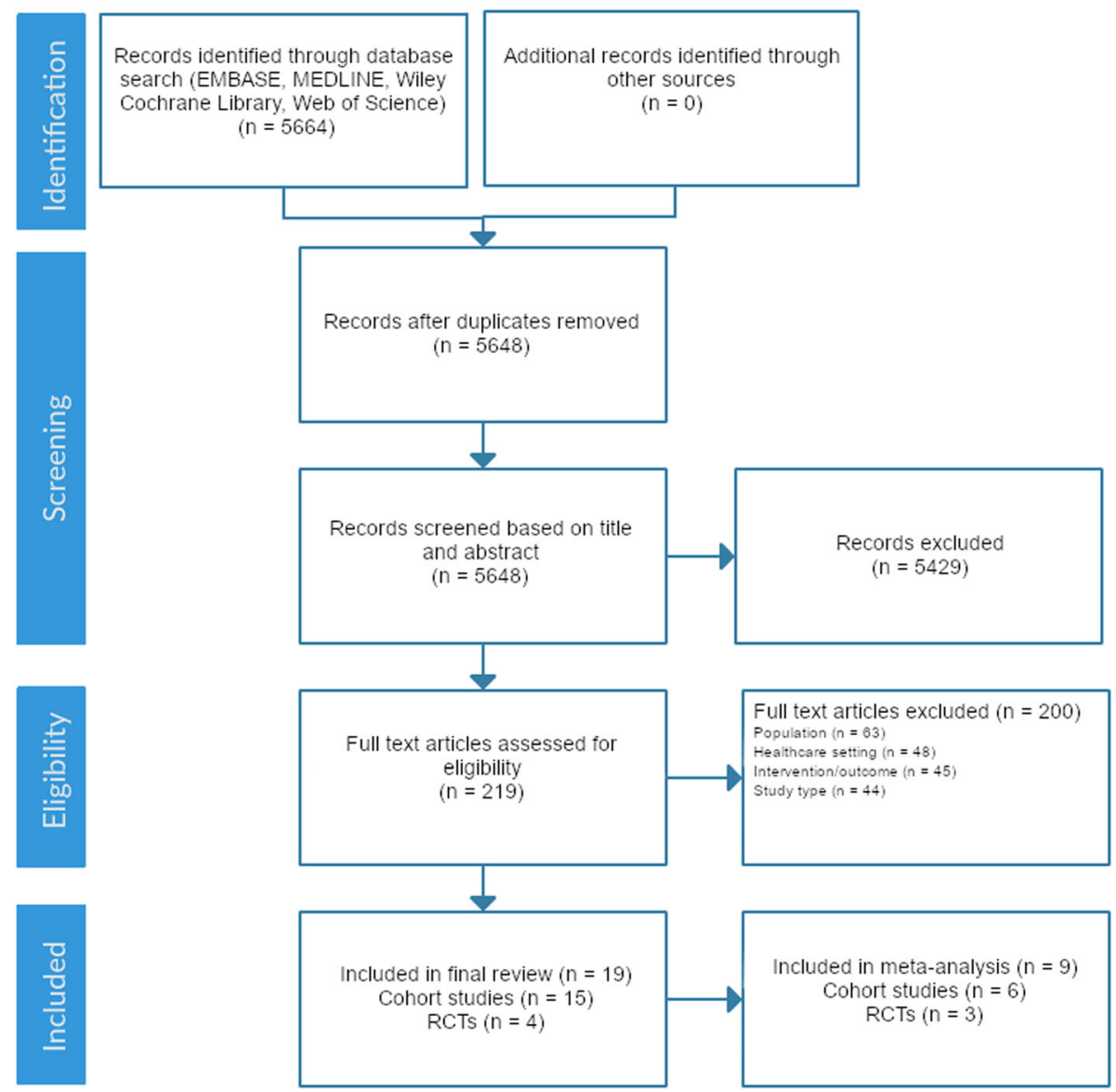

Fig. 1 Flow diagram for study assessment 
Table 2 Descriptive characteristics of included harm reduction intervention studies

\begin{tabular}{|c|c|c|c|c|c|c|c|}
\hline $\begin{array}{l}\text { Author and } \\
\text { Year of } \\
\text { Publication } \\
\end{array}$ & Setting & $\begin{array}{l}\text { \# of } \\
\text { Subjects }\end{array}$ & $\begin{array}{l}\text { Study detail } \\
\text { Inclusion criteria }\end{array}$ & $\begin{array}{l}\text { Supportive Intervention } \\
\text { Detail }\end{array}$ & $\begin{array}{l}\text { Supportive } \\
\text { Intervention } \\
\text { Category }\end{array}$ & Outcomes & $\begin{array}{l}\text { Follow-up } \\
\text { Post } \\
\text { Intervention }\end{array}$ \\
\hline \multicolumn{8}{|l|}{ Cohort studies } \\
\hline $\begin{array}{l}\text { Alburaih } \\
\text { (2018) [33] }\end{array}$ & $\mathrm{ED}$ & 314 & $\begin{array}{l}\text { Retrospective multi-centre. } \\
\text { Recurrent visits for pain. }\end{array}$ & $\begin{array}{l}\text { ED-based pain contract } \\
\text { (opioid treatment plan) }\end{array}$ & $\begin{array}{l}\text { Support for } \\
\text { pt. in pain. }\end{array}$ & \# of ED visits & 24-months \\
\hline $\begin{array}{l}\text { Alexandridis } \\
\text { (2018) [34] }\end{array}$ & $\begin{array}{l}\text { ED }+ \\
\text { Community }\end{array}$ & 7200 & $\begin{array}{l}\text { Statewide database analysis } \\
\text { Chronic pain pt. presenting } \\
\text { to ED }\end{array}$ & $\begin{array}{l}\text { Diversion control } \\
\text { Naloxone policies } \\
\text { Community education } \\
\text { Provider education } \\
\text { Support for pt. in pain } \\
\text { Hospital ED policy } \\
\text { Addiction treatment }\end{array}$ & $\begin{array}{l}\text { Diversion } \\
\text { control } \\
\text { Naloxone } \\
\text { policies } \\
\text { Community } \\
\text { education } \\
\text { Provider } \\
\text { education } \\
\text { Support for } \\
\text { pt. in pain } \\
\text { Hospital ED } \\
\text { policy } \\
\text { Addiction } \\
\text { treatment }\end{array}$ & $\begin{array}{l}\text { Overdose } \\
\text { mortality } \\
\text { Overdose- } \\
\text { related ED } \\
\text { visits }\end{array}$ & 22-months \\
\hline $\begin{array}{l}\text { Alexandridis } \\
\text { (2019) [35] }\end{array}$ & $\begin{array}{l}\text { ED + } \\
\text { Community }\end{array}$ & 7200 & $\begin{array}{l}\text { Retrospective } \\
\text { Statewide database analysis } \\
\text { Chronic pain pt. } \\
\text { Subgroup of pt. on } \\
\text { buprenorphine therapy }\end{array}$ & $\begin{array}{l}\text { Diversion control } \\
\text { Naloxone policies } \\
\text { Community education } \\
\text { Provider education } \\
\text { Support for pt. in pain } \\
\text { Hospital ED policy } \\
\text { Addiction treatment }\end{array}$ & $\begin{array}{l}\text { Diversion } \\
\text { control } \\
\text { Naloxone } \\
\text { policies } \\
\text { Community } \\
\text { education } \\
\text { Provider } \\
\text { education } \\
\text { Support for } \\
\text { pt. with pain } \\
\text { Hospital ED } \\
\text { policy } \\
\text { Addiction } \\
\text { treatment }\end{array}$ & $\begin{array}{l}\text { PDMP-derived } \\
\text { counts of } \\
\text { opioid } \\
\text { prescriptions } \\
\text { and } \\
\text { buprenorphine }\end{array}$ & 22-months \\
\hline $\begin{array}{l}\text { Allen }(2016)^{a} \\
{[36]}\end{array}$ & $\begin{array}{l}\text { ED + } \\
\text { Community }\end{array}$ & 13 & $\begin{array}{l}\text { Retrospective } \\
\text { Chart review } \\
\text { Pt with > } 360 \text { ER visits in } 12 \\
\text { months } \\
\text { Complex pain syndrome } \\
\text { Problematic substance use }\end{array}$ & $\begin{array}{l}\text { Comprehensive pain and } \\
\text { addiction strategy referral } \\
\text { from ED. }\end{array}$ & $\begin{array}{l}\text { Support for } \\
\text { pt. in pain }\end{array}$ & $\begin{array}{l}\text { \# of visits to } \\
\text { ED } \\
\text { Disposition of } \\
\text { pt. after ED } \\
\text { visit }\end{array}$ & 52-months \\
\hline $\begin{array}{l}\text { Fulton- } \\
\text { Kehoe } \\
(2015) \text { [37] }\end{array}$ & Statewide & 1809 & $\begin{array}{l}\text { Retrospective } \\
\text { Statewide database analysis } \\
\text { Pt with }>=1 \text { paid claim for } \\
\text { opioid Rx from ED. }\end{array}$ & $\begin{array}{l}\text { Statewide Guideline for best } \\
\text { practice implementation }\end{array}$ & $\begin{array}{l}\text { Statewide } \\
\text { Prescription } \\
\text { Policies }\end{array}$ & $\begin{array}{l}\text { Rates of non- } \\
\text { methadone } \\
\text { associated opi- } \\
\text { oid poisonings }\end{array}$ & 45-months \\
\hline $\begin{array}{l}\text { Ghobadi } \\
\text { (2018) [38] }\end{array}$ & ED & $19,751^{\mathrm{C}}$ & $\begin{array}{l}\text { Retrospective } \\
\text { Chart review } \\
\text { Chronic pain (>50 MEQ/d } \\
\text { for }>90 \mathrm{~d} \text { as outpatient) }\end{array}$ & $\begin{array}{l}\text { Multi-ED opioid prescribing } \\
\text { guidelines implementation }\end{array}$ & $\begin{array}{l}\text { Statewide } \\
\text { Prescription } \\
\text { Policies }\end{array}$ & $\begin{array}{l}\text { ED parenteral } \\
\text { opioids } \\
\text { ED oral } \\
\text { opioids } \\
\text { ED discharge } \\
\text { prescription } \\
\text { counts }\end{array}$ & 12-months \\
\hline $\begin{array}{l}\text { Gugelmann } \\
\text { (2013) [39] }\end{array}$ & ED & $2462^{b}$ & $\begin{array}{l}\text { Prospective } \\
\text { Pt receiving opioids in ED } \\
\text { Subgroup analysis of pt. } \\
\text { with chronic pain. }\end{array}$ & $\begin{array}{l}\text { Multifacted educational } \\
\text { program (round presentations, } \\
\text { electronic notification, formal } \\
\text { ED nursing staff education, } \\
\text { journal clubs). }\end{array}$ & $\begin{array}{l}\text { Provider } \\
\text { Education }\end{array}$ & $\begin{array}{l}\text { \# of opioid } \\
\text { discharge } \\
\text { packs } \\
\text { Change in } \\
\text { opioid } \\
\text { dispensing in } \\
\text { pt. with RF for } \\
\text { dependence }\end{array}$ & 12-months \\
\hline $\begin{array}{l}\text { Hartung } \\
\text { (2018) [40] }\end{array}$ & Statewide & N/A & $\begin{array}{l}\text { Retrospective } \\
\text { Medicaid administrative } \\
\text { claims and enrollment data } \\
\text { Pt with opioid Rx in ED. } \\
\text { Stratification by MEQ } \\
\text { dispensed. }\end{array}$ & $\begin{array}{l}\text { Prior authorization for opioid } \\
\mathrm{Rx}>120 \mathrm{mg} / \mathrm{d} \text { MEQ } \\
\text { implementation. }\end{array}$ & $\begin{array}{l}\text { Statewide } \\
\text { Prescription } \\
\text { Policies }\end{array}$ & $\begin{array}{l}\text { Probability of } \\
\text { opioid } \\
\text { prescription } \\
\text { above } 120 \mathrm{mg} \\
\text { MED }\end{array}$ & 18-months \\
\hline
\end{tabular}


Table 2 Descriptive characteristics of included harm reduction intervention studies (Continued)

\begin{tabular}{|c|c|c|c|c|c|c|c|}
\hline $\begin{array}{l}\text { Author and } \\
\text { Year of } \\
\text { Publication }\end{array}$ & Setting & $\begin{array}{l}\text { \# of } \\
\text { Subjects }\end{array}$ & $\begin{array}{l}\text { Study detail } \\
\text { Inclusion criteria }\end{array}$ & $\begin{array}{l}\text { Supportive Intervention } \\
\text { Detail }\end{array}$ & $\begin{array}{l}\text { Supportive } \\
\text { Intervention } \\
\text { Category }\end{array}$ & Outcomes & $\begin{array}{l}\text { Follow-up } \\
\text { Post } \\
\text { Intervention }\end{array}$ \\
\hline $\begin{array}{l}\text { Jurecska } \\
\text { (2012) [41] }\end{array}$ & ED & 91 & $\begin{array}{l}\text { Retrospective } \\
\text { Chart review } \\
\text { Pt with }>3 \text { ED visits in prior } \\
\text { 3-Mos or } 6 \text { or }>\text { presenta- } \\
\text { tions in } 6 \text {-Mo with chronic } \\
\text { pain (defined as pain }>6 \\
\text { Mos). }\end{array}$ & $\begin{array}{l}\text { Non-narcotic and adherence } \\
\text { rates to narcotics policy } \\
\text { implementation }\end{array}$ & $\begin{array}{l}\text { Statewide } \\
\text { Prescription } \\
\text { Policies }\end{array}$ & $\begin{array}{l}\text { Recurrent } \\
\text { visits to ED }\end{array}$ & 36-months \\
\hline $\begin{array}{l}\text { Kahler } \\
\text { (2017) [42] }\end{array}$ & $\mathrm{ED}$ & 243 & $\begin{array}{l}\text { Retrospective } \\
\text { Chart review } \\
\text { Pt with chronic pain } \\
\text { Pt with >=6 ED visits per } 12 \\
\text { Mo + at least } 1 \text { visit } \\
\text { identified as primarily } \\
\text { opioid-seeking behavior }+ \\
\text { case management for ED } \\
\text { misuse. }\end{array}$ & $\begin{array}{l}\text { Referral to free outpatient } \\
\text { taper-to-abstinence pain man- } \\
\text { agement clinic. }\end{array}$ & $\begin{array}{l}\text { Support for } \\
\text { pt. in pain }\end{array}$ & $\begin{array}{l}\text { \# of ED visits } \\
\text { \# of PDMP } \\
\text { opioid } \\
\text { prescriptions } \\
\text { \# of individual } \\
\text { opioid } \\
\text { prescribers } \\
\text { \# of diagnostic } \\
\text { tests }\end{array}$ & 12-months \\
\hline $\begin{array}{l}\text { Maughan } \\
\text { (2015) [43] }\end{array}$ & $\mathrm{ED}$ & N/A & $\begin{array}{l}\text { Retrosepctive } \\
\text { Database analysis through } \\
\text { DAWN (Drug Abuse } \\
\text { Monitoring Network) } \\
\text { All ED visits involving } \\
\text { opioid analgesic related } \\
\text { harm (abuse or accidental) }\end{array}$ & $\begin{array}{l}\text { Implementation of } \\
\text { prescription drug monitoring } \\
\text { program (PDMP) }\end{array}$ & $\begin{array}{l}\text { Electronic } \\
\text { Alert System }\end{array}$ & $\begin{array}{l}\text { Rates of ED } \\
\text { visits }\end{array}$ & 84-months \\
\hline $\begin{array}{l}\text { Olsen (2016) } \\
{[44]}\end{array}$ & ED & 46 & $\begin{array}{l}\text { Retrospective + prospective } \\
\text { Chart review } \\
\text { Pt with }>3 \mathrm{ED} \text { visits in prior } \\
\text { 6-Mo or }>6 \mathrm{ED} \text { visits in } \\
\text { prior 12-Mo for a chronic } \\
\text { painful condition. } \\
\text { Inappropriate opioid } \\
\text { prescription management }\end{array}$ & $\begin{array}{l}\text { ED opioid prescription drug } \\
\text { treatment plan in cooperation } \\
\text { with primary care provider. }\end{array}$ & $\begin{array}{l}\text { Support for } \\
\text { pt. in pain }\end{array}$ & $\begin{array}{l}\text { \# fo ED visits } \\
\text { \# of opioid } \\
\text { pills prescribed }\end{array}$ & 6-months \\
\hline $\begin{array}{l}\text { Pace (2017) } \\
{[45]}\end{array}$ & ED & 529 & $\begin{array}{l}\text { Retrospective } \\
\text { Chart review } \\
\text { Acute pain } \\
\text { Chronic pain (> } 3 \mathrm{Mo} \text { ) }\end{array}$ & $\begin{array}{l}\text { Opioid prescribing pathway } \\
\text { with framework for opioid } \\
\text { prescription }\end{array}$ & $\begin{array}{l}\text { Hospital ED } \\
\text { policy }\end{array}$ & $\begin{array}{l}\text { MEQ dose } \\
\text { administered } \\
\text { in ED } \\
\text { \# of IV/IM } \\
\text { prescrpitions } \\
\text { \# of opioid } \\
\text { prescriptions } \\
\text { at discharge }\end{array}$ & 6-months \\
\hline $\begin{array}{l}\text { Svenson } \\
\text { (2007) [46] }\end{array}$ & ED & 15 & $\begin{array}{l}\text { Prospective } \\
\text { Chart review } \\
\text { Pt with > } 10 \text { ED visits in } \\
\text { prior 12-Mo for chronic non } \\
\text { cancer pain. }\end{array}$ & $\begin{array}{l}\text { ED organized care with non- } \\
\text { opioid Rx and referral to pri- } \\
\text { mary care provider for opioid } \\
\text { management. }\end{array}$ & $\begin{array}{l}\text { Support for } \\
\text { pt. in pain }\end{array}$ & $\begin{array}{l}\text { \# of ED visits } \\
\text { \# of outpatient } \\
\text { clinic visits } \\
\text { \# of outpatient } \\
\text { opioid } \\
\text { prescriptions }\end{array}$ & 12-months \\
\hline $\begin{array}{l}\text { Whiteside } \\
\text { (2017) [47] }\end{array}$ & $\mathrm{ED}$ & 29 & $\begin{array}{l}\text { Prospective open } \\
\text { Feasibility study } \\
\text { Subgroup analysis of ED pt. } \\
\text { screened positive for risk of } \\
\text { Rx opioid misuse in prior 6- } \\
\text { Mo }\end{array}$ & $\begin{array}{l}\text { ED-LINC: Emergency } \\
\text { department longitudinal } \\
\text { integrated care. } \\
\text { Multidisciplinary case } \\
\text { management: active care } \\
\text { coordination and linkage, } \\
\text { opioid guidelines, PDMP } \\
\text { usage. }\end{array}$ & $\begin{array}{l}\text { Support for } \\
\text { pt. in pain } \\
\text { Electronic } \\
\text { Alert System } \\
\text { Hospital ED } \\
\text { policy }\end{array}$ & $\begin{array}{l}\text { Feasibility of } \\
\text { intervention } \\
\text { Substance use } \\
\text { and mental } \\
\text { health scores } \\
\text { \# of ED visits }\end{array}$ & 6-months \\
\hline \multicolumn{8}{|c|}{ Randomized Controlled Trials } \\
\hline $\begin{array}{l}\text { Murphy } \\
(2017)^{d}[48]\end{array}$ & $\mathrm{ED}$ & 165 & $\begin{array}{l}\text { Multi-centric } \\
\text { Non-blinded } \\
\text { Pt with } 5 \text { or }>\text { ED visits in } \\
\text { prior } 12 \text {-Mo with }>\text { pain } \\
\text { complaints or drug-seeking } \\
\text { behavior. } \\
\text { ED presentation }>50 \% \\
\text { related to pain. }\end{array}$ & $\begin{array}{l}\text { Multidisciplinary case } \\
\text { management with organized } \\
\text { follow-up by case manager. }\end{array}$ & $\begin{array}{l}\text { Support for } \\
\text { pt. in pain }\end{array}$ & $\begin{array}{l}\text { Total } \\
\text { treatment cost } \\
\text { differential }\end{array}$ & 12-months \\
\hline
\end{tabular}


Table 2 Descriptive characteristics of included harm reduction intervention studies (Continued)

\begin{tabular}{|c|c|c|c|c|c|c|c|}
\hline $\begin{array}{l}\text { Author and } \\
\text { Year of } \\
\text { Publication }\end{array}$ & Setting & $\begin{array}{l}\text { \# of } \\
\text { Subjects }\end{array}$ & $\begin{array}{l}\text { Study detail } \\
\text { Inclusion criteria }\end{array}$ & $\begin{array}{l}\text { Supportive Intervention } \\
\text { Detail }\end{array}$ & $\begin{array}{l}\text { Supportive } \\
\text { Intervention } \\
\text { Category }\end{array}$ & Outcomes & $\begin{array}{l}\text { Follow-up } \\
\text { Post } \\
\text { Intervention }\end{array}$ \\
\hline & & & $\begin{array}{l}\text { Economic evaluation (same } \\
\text { cohort as Neven 2016) }\end{array}$ & & & & \\
\hline $\begin{array}{l}\text { Neven } \\
(2016)[49]\end{array}$ & $\mathrm{ED}$ & 165 & $\begin{array}{l}\text { Multi-centric } \\
\text { Non-blinded } \\
\text { Pt with } 5 \text { or > ED visits in } \\
\text { prior } 12 \text {-Mo with > pain } \\
\text { complaints or drug-seeking } \\
\text { behavior. } \\
\text { ED presentation > 50\% } \\
\text { related to pain. }\end{array}$ & $\begin{array}{l}\text { Multidisciplinary case } \\
\text { management with organized } \\
\text { follow-up by case manager. }\end{array}$ & $\begin{array}{l}\text { Support for } \\
\text { pt. in pain }\end{array}$ & $\begin{array}{l}\text { \# of ED visits } \\
\text { Odds of } \\
\text { receiving an } \\
\text { opioid } \\
\text { prescription at } \\
\text { ED discharge } \\
\text { MEQ of opioid } \\
\text { dispensed }\end{array}$ & 12-months \\
\hline $\begin{array}{l}\text { Rathlev } \\
\text { (2016) [50] }\end{array}$ & $\mathrm{ED}$ & 40 & $\begin{array}{l}\text { Multi-centric } \\
\text { Non-blinded } \\
\text { Pt with } 4 \text { or }>\text { ED visits in } \\
\text { prior } 12-\text { Mo with opioid use } \\
\text { disorder (OUD) identified } \\
\text { via SMS billing codes } \\
\text { ED presentation related to } \\
\text { acute pain. }\end{array}$ & $\begin{array}{l}\text { Multidisciplinary case } \\
\text { management development }\end{array}$ & $\begin{array}{l}\text { Support for } \\
\text { pt. in pain }\end{array}$ & $\begin{array}{l}\text { MEQ } \\
\text { prescribed at } \\
\text { discharge } \\
\text { MEQ } \\
\text { administered } \\
\text { in ED or } \\
\text { inpatient } \\
\text { Total medical } \\
\text { charges } \\
\text { \# of ED visits } \\
\text { \# of ED visits } \\
\text { with advanced } \\
\text { imaging } \\
\text { \# of inpatient } \\
\text { admission }\end{array}$ & 12-months \\
\hline $\begin{array}{l}\text { Ringwalt } \\
\text { (2015) [51] }\end{array}$ & ED & 411 & $\begin{array}{l}\text { Pt with } 11 \text { or }>\text { ED visits in } \\
\text { prior } 12-\text { Mo and chronic } \\
\text { noncancer pain determined } \\
\text { via chart \& Rx review }\end{array}$ & $\begin{array}{l}\text { Care linkage to primary care } \\
\text { provider with plan for non- } \\
\text { opioid based pain } \\
\text { management. }\end{array}$ & $\begin{array}{l}\text { Support for } \\
\text { pt. in pain }\end{array}$ & $\begin{array}{l}\text { \# of } \\
\text { prescriptions } \\
\text { received from } \\
\text { ED. } \\
\text { \# of ED visits }\end{array}$ & 12-months \\
\hline
\end{tabular}

${ }^{\mathrm{a}}$ Abstract only. ${ }^{\mathrm{b}}$ Subgroup analysis of pt. on opioids at ER presentation (pre=1512 and post=950). ${ }^{\mathrm{C}}$ Subgroup of chronic opioid use pt. pre + post. ${ }^{\mathrm{d}}$ Murphy (2017) [48] is an economic evaluation of the population and harm reduction strategy studied in Neven (2016) [49]. MINI Mini-International Neuropsychiatric Interview as per DSM-IV criteria, Rx prescriptions, MME Morphine milliequivalents (synonymous with mean morphine equivalent / MEQ), ED Emergency department, PCP Primary care provider, RF Risk factors, \# Number, Pt pt., Mo Months, $d$ Days. All studies listed were compared to usual care as defined as standard practice in the institution

See supplementary appendix 1 for full references

meta-analysis assessed outcomes for the "supports for patients in pain" supportive intervention. The specific interventions included: care linkage to primary care provider with non-opioid ED intervention plan $(n=2)$ and ED opioid intervention plan $(n=1)$, isolated opioid ED intervention plan $(n=2)$, comprehensive pain and addiction strategy referral $(n=2)$ and multidisciplinary case management $(n=3)$. Two outcomes (number of ED visits and number of ED discharge opioid prescriptions) were meta-analyzed, representing 1030 patients. The ED visits outcome refers to total number of visits to the ED after implementation of the strategy and was assessed in 9 studies (6 cohort, 3 RCTs) representing 1030 patients. The ED discharge opioid prescriptions outcome refers to the number of prescriptions dispensed at discharge of the patients from the ED and was assessed in 3 studies (3 RTCs) representing 308 patients. A significant reduction in number of ED visits for cohort studies (ratio of means 0.36 , 95\% with CI [0.20-0.62], $\mathrm{I}^{2}=87 \%$ ) and for RCTs (ratio of means $0.71,95 \%$ with CI [0.61-0.82], $\mathrm{I}^{2}=$ $0 \%)$ was apparent. A significant reduction in number of
ED discharge opioid prescriptions was noted for RCTs (ratio of means $0.34,95 \%$ with CI [0.14-0.82], $\mathrm{I}^{2}=78 \%$ ). No patient-centered outcomes could be meta-analyzed. The studies duration ranged from 6 to 52 months.

\section{Discussion}

This systematic review identified 11 supportive strategies for patients on long-term prescription opioids presenting to an ED with complications related to their opioid therapy. A pooled analysis of outcomes for "support for patients in pain" showed a clinically important decrease in the number of ED visits and ED discharge opioid prescriptions. Other supportive strategies could not be analyzed in a rigorous fashion and may be considered by healthcare providers until additional evidence becomes available.

Opioid use is an important and increasing problem in the US and Canada. Multiple supportive strategies for acute healthcare settings have been developed and studied, but the evidence had not been collated for an assessment of their impact. Most of the supportive strategies 
Table 3 Quality assessment of the studies included using the Newcastle-Ottawa Scale (NOS)

\begin{tabular}{|c|c|c|c|c|c|c|c|}
\hline Author & $\begin{array}{l}\text { Representativeness } \\
\text { of exposed cohort }\end{array}$ & $\begin{array}{l}\text { Selection of } \\
\text { non-exposed } \\
\text { cohort }\end{array}$ & $\begin{array}{l}\text { Ascertainment } \\
\text { of harm } \\
\text { reduction }\end{array}$ & $\begin{array}{l}\text { Outcome of } \\
\text { interest absent at } \\
\text { start of study }\end{array}$ & $\begin{array}{l}\text { Comparability } \\
\text { of cohorts }\end{array}$ & $\begin{array}{l}\text { Assessment of } \\
\text { outcome with } \\
\text { independency }\end{array}$ & $\begin{array}{l}\text { Adequacy of } \\
\text { follow-up } \\
\text { length }\end{array}$ \\
\hline $\begin{array}{l}\text { Alburaih } \\
\text { (2018) [33] }\end{array}$ & * & & * & * & ** & * & * \\
\hline $\begin{array}{l}\text { Alexandridis } \\
\text { (2017) [11] }\end{array}$ & * & * & * & * & & * & * \\
\hline $\begin{array}{l}\text { Alexandridis } \\
\text { (2018) [34] }\end{array}$ & * & * & * & * & & * & * \\
\hline $\begin{array}{l}\text { Allen (2016) } \\
{[36]}\end{array}$ & * & * & * & * & & * & * \\
\hline $\begin{array}{l}\text { Fulton- } \\
\text { Kehoe } \\
(2015) \text { [37] }\end{array}$ & * & * & * & * & * & * & * \\
\hline $\begin{array}{l}\text { Ghobadi } \\
\text { (2018) [38] }\end{array}$ & $*$ & * & * & * & & * & * \\
\hline $\begin{array}{l}\text { Gugelmann } \\
\text { (2013) [39] }\end{array}$ & $*$ & * & * & & & * & * \\
\hline $\begin{array}{l}\text { Hartung } \\
\text { (2018) [40] }\end{array}$ & * & * & * & * & $*$ & * & * \\
\hline \multicolumn{8}{|l|}{$\begin{array}{l}\text { Jurecska } \\
\text { (2012) [41] }\end{array}$} \\
\hline $\begin{array}{l}\text { Kahler } \\
\text { (2017) [42] }\end{array}$ & $*$ & * & * & * & $* *$ & * & * \\
\hline $\begin{array}{l}\text { Maughan } \\
\text { (2015) [43] }\end{array}$ & * & * & * & * & & * & \\
\hline $\begin{array}{l}\text { Olsen (2016) } \\
\text { [44] }\end{array}$ & $*$ & * & * & * & & * & * \\
\hline $\begin{array}{l}\text { Pace (2017) } \\
{[45]}\end{array}$ & * & * & * & * & & * & * \\
\hline $\begin{array}{l}\text { Svenson } \\
\text { (2007) [46] }\end{array}$ & * & * & * & * & & * & * \\
\hline $\begin{array}{l}\text { Whiteside } \\
\text { (2017) [47] }\end{array}$ & * & * & * & * & & * & \\
\hline
\end{tabular}

A maximum score of 9 is possible. A score of 0 to 3 represent low quality; 4 to 6 represent moderate quality; 7 to 9 represent high quality.

identified were from small, single center studies, and were too heterogeneous to be meta-analyzed or were infrequently studied (Table 5 ). While most reported positive results, a number of these are single-center studies with a small number of patients. These small studies often lack the scientific rigor or external validity to allow meaningful interpretation in a larger context, and to support widespread changes in practice [52].

We identified multiple studies with enough data to perform a meta-analysis for outcomes of the "support for patient in pain" strategy (Table 5). These studies were chosen due to their similarity in the coordinated

Table 4 Quality assessment of randomized controlled trials using the Cochrane Risk of Bias Tool

\begin{tabular}{|c|c|c|c|c|c|c|c|c|}
\hline Author & $\begin{array}{l}\text { Random sequence } \\
\text { generation }\end{array}$ & $\begin{array}{l}\text { Allocation } \\
\text { concealement }\end{array}$ & $\begin{array}{l}\text { Selective } \\
\text { reporting }\end{array}$ & $\begin{array}{l}\text { Other } \\
\text { bias }\end{array}$ & $\begin{array}{l}\text { Blinding of } \\
\text { participants }\end{array}$ & $\begin{array}{l}\text { Blinding of } \\
\text { outcome }\end{array}$ & $\begin{array}{l}\text { Incomplete } \\
\text { outcome data }\end{array}$ & $\begin{array}{l}\text { Final risk } \\
\text { of bias }\end{array}$ \\
\hline $\begin{array}{l}\text { Rathlev } \\
\text { (2016) [50] }\end{array}$ & Low & Low & Low & Low & High & Unclear & Low & High \\
\hline $\begin{array}{l}\text { Neven } \\
\text { (2016) [49] }\end{array}$ & Low & Low & Low & Low & High & Low & Low & Mod \\
\hline $\begin{array}{l}\text { Murphy } \\
\text { (2017) [48] }\end{array}$ & Low & Low & Low & Low & High & Low & Low & Mod \\
\hline $\begin{array}{l}\text { Ringwalt } \\
\text { (2015) [51] }\end{array}$ & Low & High & Low & Low & High & Low & Low & Mod \\
\hline
\end{tabular}


Table 5 Supportive interventions identified

\begin{tabular}{|c|c|c|c|}
\hline $\begin{array}{l}\text { Harm Reduction } \\
\text { strategy }\end{array}$ & General Definition & Cohort & RCTs \\
\hline $\begin{array}{l}\text { Support for patients in } \\
\text { pain }\end{array}$ & Support groups, case management and pain clinic vetting and referrals. & 8 & 4 \\
\hline Hospital ED policy & $\begin{array}{l}\text { Local practices to limit ED or inpatient OA prescribing and checking prescription drugs monitoring } \\
\text { programs prior to prescribing }\end{array}$ & 4 & \\
\hline Electronic alert system & Systems that alert providers to possible opioid abuse situations without mandating their use & 4 & \\
\hline Provider education & Education of medical professionals in chronic pain treatment & 3 & \\
\hline $\begin{array}{l}\text { Statewide prescription } \\
\text { policies }\end{array}$ & Practices or wide-ranging regulations to limit OA prescription within a legislative territory & 3 & \\
\hline Addiction treatment & Opioid agonist therapies and policies supporting their use & 2 & \\
\hline Community education & Promotion of public awareness of prescription opioid overdose & 2 & \\
\hline Diversion control & Removal of unused medications and training of local law enforcement with OA diversion & 2 & \\
\hline Naloxone policies & Promotion of the adoption of policies to disseminate the opioid antagonist naloxone to opioid users & 2 & \\
\hline
\end{tabular}

care models used and the target populations. There was a clinically important decrease in system-related outcomes of ED visits and ED discharge opioid prescriptions for this strategy. For both outcomes, 3 RCTs were included with the most compelling data for ED visits due statistical significance and uniform data $\left(\mathrm{I}^{2} 0 \%\right)$, while ED discharge opioid prescriptions were significant but showed substantial heterogeneity ( $\left.\mathrm{I}^{2} 87 \%\right)$. The ED visits outcome was also supported by the meta-analysis of cohort studies that all trended in the same direction despite substantial heterogeneity ( $\left.\mathrm{I}^{2} 87 \%\right)$. As discussed above, "support for patients in pain" represent an aggregation of strategies individualized to a specific patient's needs, which is widely different from other supportive interventions. These types of interventions are of course more resource intensive. Overall, the evidence demonstrates that the costs of treatment for opioid misuse and abuse are offset by the reduced health care costs [53,
54]. Murphy et al. provides the only economic analysis that indicates similar findings [48] and may support an economic incentive to their use in long-term opioid users without opioid use disorder. Furthermore, these interventions have clear evidence and support for patients with chronic non-cancer pain and opioid use disorder [55-57]. They are accordingly recommended by different international guidelines with recommendations [58]. Unfortunately, our findings are more complex in terms of interpretation by the nature of the outcomes used. Indeed, across all studies, there were only four instances of patient-related outcomes being evaluated. In these cases, the decrease in system-related outcomes were associated with unfavorable patient-related outcomes. Fulton-Kehoe indeed showed an increase in methadone poisonings as the number of opioid prescriptions and poisonings decreased [37] Alexandridis et al. was the only study with a favorable patient-related

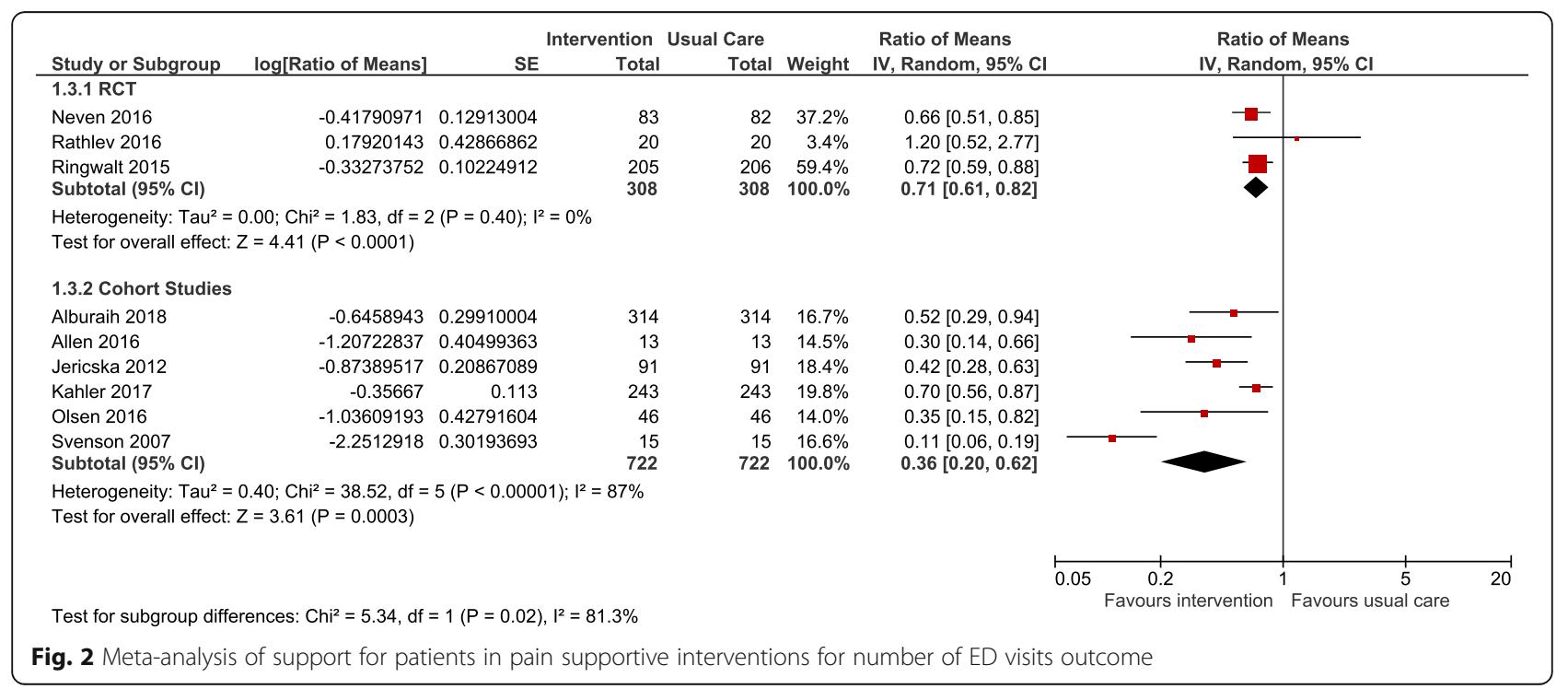




\begin{tabular}{|c|c|c|c|c|c|c|c|}
\hline Study or Subgroup & log[Ratio of Means] & SE & Weight & $\begin{array}{l}\text { Ratio of Means } \\
\text { IV, Random, } 95 \% \mathrm{Cl}\end{array}$ & $\begin{array}{r}\text { Ratio of } \\
\text { IV, Rando }\end{array}$ & I Means & \\
\hline Neven 2016 & -1.63760879 & 0.32995213 & $36.5 \%$ & $0.19[0.10,0.37]$ & - & & \\
\hline Rathlev 2016 & -1.238 & 0.69 & $21.8 \%$ & $0.29[0.07,1.12]$ & . & & \\
\hline Ringwalt 2015 & -0.48550782 & 0.20507756 & $41.6 \%$ & $0.62[0.41,0.92]$ & $\longrightarrow-$ & & \\
\hline Total $(95 \% \mathrm{Cl})$ & & & $100.0 \%$ & $0.34[0.14,0.82]$ & & & \\
\hline \multicolumn{4}{|c|}{$\begin{array}{l}\text { Heterogeneity: } \mathrm{Tau}^{2}=0.44 ; \mathrm{Chi}^{2}=9.16, \mathrm{df}=2(P=0.01) ; I^{2}=78 \% \\
\text { Test for overall effect: } Z=2.40(P=0.02)\end{array}$} & & $\begin{array}{cccc}0.1 & 0.2 & 0.5 & 1 \\
& \text { Favours intervention }\end{array}$ & $\begin{array}{cc}2 & 5 \\
\text { Favours usual care }\end{array}$ & 10 \\
\hline
\end{tabular}

outcome, demonstrating lower overdose mortality related to healthcare professional education, but as a whole did not change the rate of ED visits [11]. This highlights concerns by experts that harm reduction strategies that focus on decreasing opioid prescriptions might actually contributed to unanticipated increases in avoidable deaths and overdoses [59] as patients seek out non-prescribed opioids to replace the previously prescribed opioids. The outcomes meta-analyzed may thus represent a poor proxy for appropriately impactful supportive strategies.

The other supportive strategies listed in Table 5 represent a combination of frequently recurring well-defined supportive strategies as well as composite terms representing supportive strategies referred to with different names across studies. This was determined through careful review of the detailed intervention performed in each study in order to reclassify them under umbrella headings. Unfortunately, precise definitions for each harm reduction strategy identified were not present in most studies. This limits our ability to both have homogeneous interventions under each harm reduction strategy. As such, based on the analysis of the interventions performed, most studies have multiple simultaneous harm reductions strategies employed. Accordingly, this limits the rigorous analysis of each harm reduction strategy independently.

In a similar fashion, there are no comparative studies of supportive strategies to inform which strategies may be superior, in which specific context, and where to direct organization and resources. Alexandridis et al. was the only study to include multiple welldifferentiated strategies but analyzed them as independent variables despite a simultaneous implementation [11]. However, identifying a superior strategy may be of limited importance, as statistical superiority does not necessarily reflect the clinical reality in these complex patients. Indeed, the most appropriate strategy depends on multiple local factors such as individual patient's specific needs and availability as well as access to resources. This highlights the complexity of assessing these process of care interventions for successful implementation and effectiveness of intervention. Such interventions may not lead to statistical or clinical significance in traditional outcomes (i.e., mortality) but have wider ranging benefits in care processes, workflow and resource optimization, as in the case and wide adoption of medical emergency teams (MET) [60].

\section{Strengths and limitations}

While this study had several important strengths (i.e., breadth of scope, rigorously pre-defined methodology stretching across several medical domains, presence of patient advisors), several important limitations warrant discussion. First, important terms (i.e., long-term medical opioid therapy, opioid 'abuse' and misuse, harm reduction strategies) were heterogeneously defined across studies and may have been a barrier to study identification. Most importantly, the supportive strategies were overall poorly defined across studies. Despite a careful analysis of the interventions to regroup or reclassify them under umbrella terms, it was difficult to clearly identify separate supportive strategies in some studies. Accordingly, these studies then often used multiple supportive strategies simultaneously, which significantly limited our ability to have a rigorous analysis. This is reflected in the meta-analysis where the most important harm reduction strategy was analyzed, acknowledging that it may not be fully separated from other minor elements of the intervention that may be classified under another umbrella term. We attempted to mitigate these factors by independent screening by two authors to ensure the inclusion of all relevant studies and appropriately classify the supportive strategies. Second, the rate of study inclusion was only $0.4 \%$. This was secondary to most identified studies either studied illicit drug use or poorly differentiated long-term opioid use without opioid disorder from opioid use disorder. We aimed to exclude opioid use disorder or abuse but were faced with a high degree of uncertainty in some cases. We thus decided to include studies only if they specifically referred to acute pain presentation in long-term opioid users even if there was mention of prescription opioid misuse, as long as there was some clear distinction between the groups. Given that there is a degree of conversion from 
appropriate use to abuse, we believe that this captures well this evolution in patients. Similarly, identifying what constitutes harm or complications from long-term opioid use proved challenging. Presentations other than overdose or without the attached opioid misuse label are often unrecognized as related to opioids. We had to assume that in the selected papers, the focus on patients being on opioid therapy means that there is a reasonable expectation that their presentation is related to opioid in some way. In our opinion, acute on chronic pain qualified as such, as it either represents hyperalgesia or under-treatment, both of which require a measured treatment approach. Third, the wide scope of some supportive strategies lead to difficult decisions for study inclusion. Indeed, a number of harm reductions were part of a package organized at a state level. It was difficult to separate the specific impact of each strategy, the impact on acute versus non-acute healthcare settings and to discern which studies dealt with patients on appropriate long-term opioid therapy. In these situations, we opted to include these state level studies if there was a welldescribed significant proportion of long-term opioid users, and if number of acute healthcare presentations was an outcome of interest. We do acknowledge that these studies reflect a very heterogeneous group in a lot of instances and limit the validity of the findings. This is not reflected well in the quality assessment of the cohort study who are technically for the most part of moderate to high methodological quality. The RCTs are for their part paradoxically at moderate to high risk of bias due to their design but represent a more homogeneous population. Fourth, most identified studies were from the US, limiting the generalizability of our findings to other jurisdictions that may have different policies and context that affect the outcomes of the identified supportive strategies. This is not surprising as the opioid epidemic was first recognized in the US, and many findings in the US are applicable across Canada and other high-income countries [1]. Finally, while we decided to include studies from 1996, all of the studies included are from the last 15 years. This is likely explained by the delayed recognition of the public health crisis from the opioid epidemic.

\section{Future directions}

Our systematic review revealed that most of the studies have targeted patients presenting to the ED, with very little data on inpatient supportive strategies. This knowledge gap is reflected in the most recent Canadian guidelines for opioid use for chronic non-cancer pain, which do not address acute admissions in this population [58]. These guidelines do reflect the importance of a multidisciplinary approach in the chronic non-cancer pain population, which would be similar to the "supports for patient in pain" harm reductions strategy. Studying this harm reduction strategy for non-ED acute healthcare settings would strengthen the current body of evidence. Importantly, studying these strategies using patientrelated outcomes such as mortality, quality of life and pain is of paramount importance, as opioid prescriptions and ED visits appear to be poor or misleading surrogate endpoints. Future policy work informed by these results would lead to better resource utilization through a shift from reactionary processes (i.e., ED visits) to preventative strategies that prevent acute healthcare presentations.

\section{Conclusion}

We identified 9 supportive strategies for patients chronically prescribed opioids presenting to acute healthcare. The only harm reduction strategy that showed evidence of efficacy what "support for patients in pain" with clinically important decrease in the number of ED visits and ED discharge opioid prescriptions. Unfortunately, other supportive strategies were not evaluated in a rigorous fashion and may be considered by healthcare providers until additional evidence becomes available. These strategies have been studied almost exclusively in ED patients, and data on inpatient harm reduction is lacking and requires further study.

\section{Supplementary Information}

The online version contains supplementary material available at https://doi. org/10.1186/s12873-020-00398-9.

Additional file 1. Variables extracted from included articles.

Additional file 2. References of included studies.

\section{Abbreviations \\ ED: Emergency Department; ICU: Intensive Care Unit; RCT: Randomized Control Trial; MET: Medical Emergency Team}

Acknowledgments

Not applicable.

Authors' contributions

JD1 was responsible for the manuscript preparation. JD1 and OGR were responsible for finalizing the manuscript. RF developed the search strategy in consultation with JD1 and OGR and conducted the search. BV provided statistical methodology and analysis. SMB and OGR conceived the project, and all authors (JD1, JG, SS, KD, FM, CK, LM, RM1, JD2, HC, JM, KR, DL, RM2, $J D, J G, S M B, O G R, M S$ ) provided critical revision of the final manuscript. OGR will guarantee the content of the review. All authors read and approved the final manuscript.

\section{Funding}

This project is supported by the Alberta Strategy for Patient Oriented Research (SPOR) SUPPORT Unit Knowledge Translation program. SPOR provided support in the form of a research librarian, statistician and project coordinator.

Availability of data and materials

The data generated or analysed during this study is available from the corresponding author upon reasonable request. 


\section{Ethics approval and consent to participate}

Not applicable.

\section{Consent for publication}

Not applicable.

\section{Competing interests}

SMB is supported by a Canada Research Chair in Critical Care Nephrology. SS has received advisory board fees from Daiichi Sankyo, Inc. SS is a specialist in occupational medicine and some of the patients he assesses have painful conditions.

\section{Author details}

'Department of Critical Care Medicine, Faculty of Medicine and Dentistry, University of Alberta, 8440112 St. NW, Critical Care Medicine 2-124E Clinical Sciences Building, Edmonton, Alberta T6G 2B7, Canada. ${ }^{2}$ School of Medicine, Faculty of Medicine and Dentistry, University of Alberta, Edmonton, Alberta, Canada. ${ }^{3}$ Division of Preventive Medicine, Department of Medicine, University of Alberta, 5-30 University Terrace, 8303 - 112 St NW, Edmonton, Alberta T6G 2T4, Canada. ${ }^{4}$ Department of Emergency Medicine, University of Alberta, 2J2.00 WC Mackenzie Health Sciences Centre, 8440112 St NW, Edmonton, Alberta T6G 2R7, Canada. ${ }^{5}$ Departments of Psychiatry and Pediatrics, University of Calgary, Strategic Clinical Network for Addictions and Mental Health 2888 Shaganappi Trail NW Calgary, Calgary, Alberta T3B 6A8, Canada. ${ }^{6}$ Department of Family Medicine, University of Alberta, Suite 205 College Plaza, 8215112 St NW, Edmonton, Alberta T6G 2C8, Canada. 'Department of Family Medicine, Calgary Chronic Pain Center 1820 Richmond Road SW Calgary, Calgary, Alberta T2T 5C7, Canada. ${ }^{8}$ Department of Anesthesia, University of Ottawa, Ottawa, Ontario, Canada. ${ }^{9}$ Department of Medicine, University of Ottawa, Ottawa, Ontario, Canada. ${ }^{10}$ Department of Anesthesia and Pain Management, Toronto General Hospital, University Health Network, Ottawa, Ontario, Canada. ${ }^{11}$ Transitional Pain Program, Toronto General Hospital, University Health Network, Ottawa, Ontario, Canada. ${ }^{12}$ Department of Critical Care Medicine, Queen's University, Kingston, Ontario, Canada. ${ }^{13}$ Addiction \& Mental Health Strategic Clinical Network, Alberta Health Services, Edmonton, Alberta, Canada. ${ }^{14}$ Department of Psychiatry, University of Alberta, Edmonton, Alberta, Canada. ${ }^{15}$ Department of Psychiatry, University of Calgary, Calgary, Canada. ${ }^{16}$ Alberta Research Centre for Health Evidence, Department of Pediatrics, University of Alberta, Alberta SPOR SUPPORT Unit KT Platform, 4-486D Edmonton Clinical Health Academy, 11405 - 87 Avenue, Edmonton, Alberta T6G 1C9, Canada. ${ }^{17}$ Knowledge Translation Platform, Alberta SPOR SUPPORT Unit Department of Pediatrics, University of Alberta, 362-B Heritage Medical Research Centre (HMRC), Edmonton, Canada. ${ }^{18}$ Primary Health Care Information Network, Edmonton, Alberta, Canada. ${ }^{19}$ Critical Care Strategic Clinical Network, Foothills Medical Centre, ICU Administration - Ground Floor, McCaig Tower, 3134 Hospital Drive, Calgary, Alberta T2N 2T9, Canada.

Received: 27 April 2020 Accepted: 23 December 2020 Published online: 29 January 2021

\section{References}

1. DeWeerdt S. Tracing the US opioid crisis to its roots. Nature. 2019;573(7773): S10-2.

2. Clarke H, Bao J, Weinrib A, Dubin RE, Kahan M. Canada's hidden opioid crisis: the health care system's inability to manage high-dose opioid patients. Can Fam Physician. 2019;65(9):612-4.

3. National Academies of Sciences E, Division H and M, Policy B on HS, Abuse C on PM and RS to APO, Phillips JK, Ford MA, et al. Trends in Opioid Use, Harms, and Treatment. US: National Academies Press; 2017. [cited 2020 Feb 16]. Available from: http://www.ncbi.nlm.nih.gov/books/NBK458661/.

4. Dunn KM, Saunders KW, Rutter CM, Banta-Green CJ, Merrill JO, Sullivan MD, et al. Opioid prescriptions for chronic pain and overdose: a cohort study. Ann Intern Med. 2010;152(2):85-92.

5. Shipton EE, Shipton AJ, Williman JA, Shipton EA. Deaths from opioid overdosing: implications of coroners' inquest reports 2008-2012 and annual rise in opioid prescription rates: a population-based cohort study. Pain Ther. 2017;6(2):203-15.

6. Cannon R, Bozeman M, Miller KR, Smith JW, Harbrecht B, Franklin G, et al. The prevalence and impact of prescription controlled substance use among injured patients at a level I trauma center. J Trauma Acute Care Surg. 2014; 76(1):172-5.

7. de Wit M, Gennings C, Zilberberg M, Burnham EL, Moss M, Balster RL. Drug withdrawal, cocaine and sedative use disorders increase the need for mechanical ventilation in medical patients. Addiction. 2008;103(9):1500-8.

8. Gomes T, Mamdani MM, Paterson JM, Dhalla IA, Juurlink DN. Trends in highdose opioid prescribing in Canada. Can Fam Physician Med Fam Can. 2014; 60(9):826-32.

9. Burkes R, Pfister G, Guinn B, Cavallazzi R. Opioid overdose leading to intensive care unit admission: epidemiology and outcomes. J Crit Care. 2017;37:261

10. White AM, Hingson RW, Pan I-J, Yi H-Y. Hospitalizations for alcohol and drug overdoses in young adults ages 18-24 in the United States, 1999-2008: results from the Nationwide inpatient sample. J Stud Alcohol Drugs. 2011;72(5):774-86.

11. Alexandridis AA, McCort A, Ringwalt CL, Sachdeva N, Sanford C, Marshall SW, et al. A statewide evaluation of seven strategies to reduce opioid overdose in North Carolina. Inj Prev J Int Soc Child Adolesc Inj Prev. 20.

12. British Columbia Center on Substance Use, Health BCM of. A guideline for the clinical management of opioid use disorder; 2017. p. 1-77.

13. Kucukarslan SN, Peters M, Mlynarek M, Nafziger DA. Pharmacists on rounding teams reduce preventable adverse drug events in hospital general medicine units. Arch Intern Med. 2003;163(17):2014-8.

14. Leape LL, Cullen DJ, Clapp MD, Burdick E, Demonaco HJ, Erickson Jl, et al. Pharmacist participation on physician rounds and adverse drug events in the intensive care unit. JAMA. 1999;282(3):267-70.

15. Lynch ME, Katz J. "One size fits all" Doesn't fit when it comes to long-term opioid use for people with chronic pain. Can J Pain. 2017;1(1):2-7.

16. Morley-Forster PK, Clark AJ, Speechley M, Moulin DE. Attitudes toward opioid use for chronic pain: a Canadian physician survey. Pain Res Manag. 2003;8(4):189-94.

17. National Advisory Council on Prescription Drug Misuse. First Do No Harm: Responding to Canada's Prescription Drug Crisis (Report); 2013. p. 1-84.

18. Rudd RA, Seth P, David F, Scholl L. Increases in drug and opioid-involved overdose deaths - United States, 2010-2015. MMWR Morb Mortal Wkly Rep. 2016;65(5051):1445-52.

19. Young A, Alfred KC, Davignon PP, Hughes LM, Robin LA, Chaudhry HJ. Physician survey examining the impact of an educational tool for responsible opioid prescribing. J Opioid Manag. 2012;8(2):81-7.

20. Herzig SJ, Rothberg MB, Cheung M, Ngo LH, Marcantonio ER. Opioid utilization and opioid-related adverse events in nonsurgical patients in US hospitals. J Hosp Med. 2014;9(2):73-81.

21. Van Hook C, Burneikiene S, Tangel D, Warner B. The relationship of outpatient prescription narcotic use to the early implementation and manner of assisted ventilation in a community hospital intensive care unit Intensive Care Med Exp. 2015;3(Suppl 1):A311.

22. Rootman DB, Mustard R, Kalia V, Ahmed N. Increased incidence of complications in trauma patients cointoxicated with alcohol and other drugs. J Trauma Inj Infect Crit Care. 2007;62(3):755-8.

23. $\mathrm{WHO} \mid$ Health systems and services: the role of acute care. WHO. [cited 2020 Feb 27]. Available from: https://www.who.int/bulletin/volumes/ 91/5/12-112664/en/.

24. Higgins J, Green S, editors. Cochrane Handbook for Systematic Reviews of Interventions. The Cochrane Collaboration; 2011 [cited 2018 Oct 10]. Available from: http://www.handbook.cochrane.org.

25. Centre for Reviews Dissemination. Systematic review: CRD's guidance for undertaking reviews in health care. University of York: CRD; 2009.

26. Liberati A, Altman DG, Tetzlaff J, Mulrow C, Gøtzsche PC, loannidis JPA, et al. The PRISMA statement for reporting systematic reviews and meta-analyses of studies that evaluate healthcare interventions: explanation and elaboration. BMJ. 2009;339:b2700.

27. Stroup DF, Berlin JA, Morton SC, Olkin I, Williamson GD, Rennie D, et al. Meta-analysis of observational studies in epidemiology: a proposal for reporting. Meta-analysis Of Observational Studies in Epidemiology (MOOSE) group. In: JAMA. Atlanta, GA 30333, USA. dfs2@cdc.gov: Centers for Disease Control and Prevention; 2000. p. 2008-12.

28. Yaffe PB, Green RS, Butler MB, Witter T. Is admission to the intensive care unit associated with chronic opioid use? A 4-year follow-up of intensive care unit survivors. J Intensive Care Med. 20161:32(7):429-35.

29. Korff MV, Saunders K, Thomas Ray G, Boudreau D, Campbell C, Merrill J, et al. De facto long-term opioid therapy for noncancer pain. Clin J Pain. 2008;24(6):521-7. 
30. Wells G, Shea B, O'Connell D, Peterson J, Welch V, Losos M, et al. The Newcastle-Ottawa scale (NOS) for assessing the quality of nonrandomised studies in meta-analyses. The Ottawa Hospital Research Institute; Available from: http://www.ohri.ca/programs/clinical_epidemiology/oxford.asp.

31. Rewa OG, Villeneuve P-M, Lachance P, Eurich DT, Stelfox HT, Gibney RTN, et al. Quality indicators of continuous renal replacement therapy (CRRT) care in critically ill patients: a systematic review. Intensive Care Med. 2017; 43(6):750-63.

32. Friedrich JO, Adhikari NK, Beyene J. The ratio of means method as an alternative to mean differences for analyzing continuous outcome variables in meta-analysis: a simulation study. BMC Med Res Methodol. 2008;8(1):32.

33. Alburaih A, Witting MD. Effectiveness of a Rural Emergency Department (ED)-Based Pain Contract on ED Visits Among ED Frequent Users. J Emerg Med. 2018;55(3):327-332.e1.

34. Alexandridis AA, McCort A, Ringwalt CL, Sachdeva N, Sanford C, Marshall SW, et al. A statewide evaluation of seven strategies to reduce opioid overdose in North Carolina. Inj Prev. 2018;24(1):48-54.

35. Alexandridis AA, Dasgupta N, McCort AD, Ringwalt CL, Rosamond WD, Chelminski PR, et al. Associations between implementation of Project Lazarus and opioid analgesic dispensing and buprenorphine utilization in North Carolina, 2009-2014. Inj Epidemiol. 2019;6 Available from: https://www.ncbi.nlm.nih.gov/pmc/articles/PMC633986 7/. Cited 1 July 2019.

36. Allen MA. P008: addressing chronic pain and problematic substance use of opioids in the emergency department: can a comprehensive framework for care work? Can J Emerg Med. 2016;18(S1):S81.

37. Fulton-Kehoe D, Sullivan M, Turner J, Garg R, Bauer A, Wickizer T, et al. Opioid poisonings in Washington state Medicaid: trends, dosing, and guidelines. Med Care. 2015;53(8):679-85.

38. Ghobadi A, Van Winkle PJ, Menchine M, Chen Q, Huang BZ, Sharp AL. Reduction of parenteral opioid use in community emergency departments following implementation of treatment guidelines. Acad Emerg Med. 2018.

39. Gugelmann H, Shofer FS, Meisel ZF, Perrone J. Multidisciplinary intervention decreases the use of opioid medication discharge packs from 2 urban EDs. Am J Emerg Med. 2013;31(9):1343-8.

40. Hartung DM, Kim H, Ahmed SM, Middleton L, Keast S, Deyo RA, et al. Effect of a high dosage opioid prior authorization policy on prescription opioid use, misuse, and overdose outcomes. Subst Abus. 2018;39(2):239-46.

41. Jurecska DE, Peterson MA, Turgensen JN, Florea J. Pain: The continuing epidemic. J Pain Manage. 2012;5(3)(273-278):1.

42. Kahler Z, Musey P, Schaffer J, Johnson A, Strachan C, Shufflebarger C. Effect of a "No Superuser Opioid Prescription" policy on ED visits and statewide opioid prescription. West J Emerg Med. 2017;18(5):894-902.

43. Maughan BC, Bachhuber MA, Mitra N, Starrels JL. Prescription monitoring programs and emergency department visits involving opioids, 2004-2011. Drug Alcohol Depend. 2015;156:282-8.

44. Olsen JC, Ogarek JL, Goldenberg EJ, Sulo S. Impact of a chronic pain protocol on emergency department utilization. Bird SB, editor. Acad Emerg Med. 2016;23(4):424-32.

45. Pace $C$, Shah $S$, Zhang AX, Zosel AE. Impact of a chronic pain management pathway on opioid administration and prescribing in an Emergency Department. Clin Toxicol. 2017;56(8):744-50.

46. Svenson JE, Meyer TD. Effectiveness of nonnarcotic protocol for the treatment of acute exacerbations of chronic nonmalignant pain. Am J Emerg Med. 2007;25(4):445-9.

47. Whiteside LK, Darnell D, Jackson K, Wang J, Russo J, Donovan DM, et al. Collaborative care from the emergency department for injured patients with prescription drug misuse: An open feasibility study. J Subst Abus Treat. 2017:82:12-21.

48. Murphy SM, Howell D, McPherson S, Grohs R, Roll J, Neven D. A randomized controlled trial of a citywide emergency department carecoordination program to reduce prescription opioid-related visits: an economic evaluation. J Emerg Med. 2017;53(2):186-94.

49. Neven D, Paulozzi LJ, Howell D, McPherson S, Murphy SM, Grohs B, et al. A randomized controlled trial of a citywide emergency department care coordination program to reduce prescription opioid related ED visits. J Emerg Med. 2016;51(5):498-507.

50. Rathlev N, Almomen R, Deutsch A, Smithline $\mathrm{H}$, Li H, Visintainer $\mathrm{P}$. Randomized controlled trial of electronic care plan alerts and resource utilization by high frequency emergency department users with opioid use disorder. West J Emerg Med. 2016;17(1):28-34.
51. Ringwalt C, Shanahan M, Wodarski S, Jones J, Schaffer D, Fusaro A, et al. A randomized controlled trial of an emergency department intervention for patients with chronic noncancer pain. J Emerg Med. 2015;49(6):974-83.

52. Bellomo R, Warrillow SJ, Reade MC. Why we should be wary of single-center trials. Crit Care Med. 2009;37(12):3114-9.

53. Krumova EK, Bennemann P, Kindler D, Schwarzer A, Zenz M, Maier C. Low pain intensity after opioid withdrawal as a first step of a comprehensive pain rehabilitation program predicts long-term nonuse of opioids in chronic noncancer pain. Clin J Pain. 2013;29(9):760-9.

54. Hooten WM, Shi Y, Gazelka HM, Warner DO. The effects of depression and smoking on pain severity and opioid use in patients with chronic pain. Pain. 2011;152(1):223-9.

55. Wickizer TM, Krupski A, Stark KD, Mancuso D, Campbell K. The effect of substance abuse treatment on Medicaid expenditures among general assistance welfare clients in Washington state. Milbank Q. 2006:84(3):555-76.

56. Wickizer TM, Mancuso D, Huber A. Evaluation of an innovative Medicaid health policy initiative to expand substance abuse treatment in Washington state. Med Care Res Rev. 2012;69(5):540-59.

57. Zarkin GA, Dunlap $L$, Homsi G. The substance abuse services cost analysis program (SASCAP): a new method for estimating drug treatment services costs. Eval Program Plann. 2004;27(1):35-43.

58. Busse JW, Craigie S, Juurlink DN, Buckley DN, Wang L, Couban RJ, et al. Guideline for opioid therapy and chronic noncancer pain. CMAJ. 2017; 189(18):E659-66.

59. Clarke H, Bao J, Weinrib A, Dubin RE, Kahan M. Canada's hidden opioid crisis: the health care system's inability to manage high-dose opioid patients: fallout from the 2017 Canadian opioid guidelines. Can Fam Physician. 2019;65(9):612-4.

60. Hillman K, Chen J, Cretikos M, Bellomo R, Brown D, Doig G, et al. Introduction of the medical emergency team (MET) system: a clusterrandomised controlled trial. Lancet Lond Engl. 2005;365(9477):2091-7.

\section{Publisher's Note}

Springer Nature remains neutral with regard to jurisdictional claims in published maps and institutional affiliations.
Ready to submit your research? Choose BMC and benefit from:

- fast, convenient online submission

- thorough peer review by experienced researchers in your field

- rapid publication on acceptance

- support for research data, including large and complex data types

- gold Open Access which fosters wider collaboration and increased citations

- maximum visibility for your research: over $100 \mathrm{M}$ website views per year

At $\mathrm{BMC}$, research is always in progress.

Learn more biomedcentral.com/submissions 